\title{
Nourriture(s) dans l'Odyssée: fruits, légumes et les oies de Pénélope
}

\author{
Teodoro Rennó Assunção \\ FALE-UFMG \\ teorenno@letras.ufmg.br
}

RESUMO:

Este artigo apresenta e comenta três tipos de comida possível (as frutas, os legumes e os gansos de Penélope) que na Odisséia jamais compõem o cardápio de uma refeição tradicional no mundo humano (ou seja: a carne assada de animais sacrificáveis, o pão de trigo ou de ceváda, e o vinho misturado à água), mas que poderiam ser percebidos enquanto tais pelo auditório, constituindo assim no próprio poema - ainda que de uma maneira indireta - um repertório alimentar mais largo que, por contraste, revelaria a restrição e a escolha (muito bem marcadas) do que as personagens comem no mundo humano.

PALAVRAS-CHAVE: comida; Odisséia; frutos; legumes; os gansos de Penélope.

\section{Introduction: note méthodologique}

Essayons d'abord d'esquisser un bref tableau de la nourriture mangée ou mangeable hors du cadre normal du repas traditionnel dans l'Odyssée, c'est-à-dire du menu composé par le pain, la viande rôtie des animaux domestiques sacrifiables et le vin (melangé avec de l'eau). À l'occurrence du lait, du fromage et du miel - nos trois premiers exemples (et qui ne seront pas repris ici) - comme nourriture hors du contexte humain dans l'Odyssée (c'est-à-dire surtout dans le contexte extraordinaire des voyages merveilleux d'Ulysse ou, plus précisément, dans l'épisode du Cyclope et dans celui de Circé) on peut ajouter encore le lotus ( $\lambda \omega$ Tós), "fruit doux comme le miel"

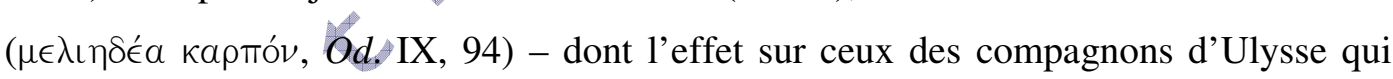
l'ont goûté suggère plutôt une sorte de drogue magique (cf. Od. IX, 95-97) - ainsi que des animaux sauvages chassés comme les 119 chèvres montagnardes (aîyas ópeokụ́ous, Od. IX, 155) dans l'île qui se situe en face du pays des Cyclopes

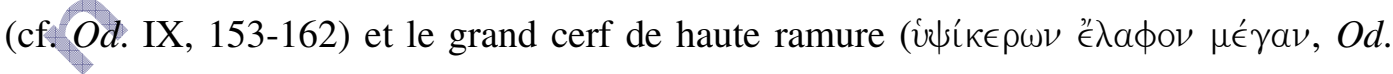
X, 158) tué et transporté par Ulysse dans l'île de Circé (cf. Od. X, 161-171; 181-184), et

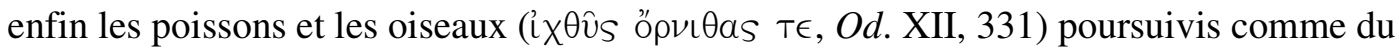

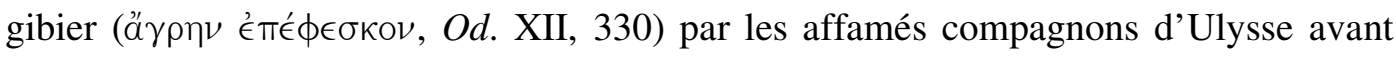
qu'ils ne chassent et tuent pour les manger les vaches sacrées du Soleil (cf. Od. XII, 353-365).

Ce répertoire quelque peu élargi (dans ce contexte extraordinaire) - auquel nous reviendrons dans la suite pour préciser quelques points - permet déjà de percevoir 
combien est retréci le menu déjà cité du contexte humain normal: le pain, la viande rôtie des animaux domestiques sacrifiables et le vin (melangé avec de l'eau). C'est ce que - à partir d'un menu grec plus varié et tardif - avait déjà bien perçu Athénée lorsque, après avoir cité les vers 138, 139 et 141 du chant I de l'Odyssée (qui définissent le pain et la viande comme nourriture homérique), il remarque à propos d'Homère:

En dehors de cela, ni dans les fêtes, ni aux noces, ni dans aucune réunion, il ne fait rien servir, quoique souvent il met en scène Agamemnon donnant à dînner aux chefs. Ni omelettes ni pâtés ou flans pas plus que gâteaux au miel ne sont par Homère servis spécialement aux rois, mais seulement ce qui devrait profiter à leur corps et à leur âme. ${ }^{1}$

Et, peu après, Athénée ajoute: "Même les prétendants, gens pleins d'insolence et adonnés aux plaisirs, ne mangent dans son poème ni poissons ni volailles ni gâteaux au miel: il supprime tant qu'il peut les artifices de la cuisine (...)".2 Et encore: "D'autre part, il ne fait même jamais servir des fruits, quoiqu'il les dise abondants et se plaise à les mentionner et à les dire perpétuellement soustraits à la mort". 3

On pourrait être tenté de savoir, par exemple, ce que les données archéologiques (et les tablettes du linéaire B) nous revèlent sur la nourriture mangée par les Grecs à l'âge du bronze ou au deuxième millénaire avant Jesus Christ. Les recherches de Kenton F. Vickery (dans Food in Early Greece), ${ }^{4}$ de Gerda Bruns (dans le chapitre 1 "Küchenwesen in mykenischen Zeit" de Archaeologia homerica. Küchenwesen und Mahlzeiten) ${ }^{5}$ et de Andrew Dalby (dans le chapitre 2 "The gardens of Alcinous" de Siren feasts $)^{6}$ indiquent en fait un répertoire alimentaire beaucoup plus varié que celui des repas normaux de l'Odyssée: d'abord le pain (même si sans levure) d'orge (dans le linéaire B: $k i$-ri-ta) ou de blé (lin. B: si-to), mais éventuellement assaisonné de coriandre (lin. B: ko-ri-ja-do-no), de céleri, de cumin (lin. B: ku-mi-no), de fénouil (lin. B: ma-ra-tu-wo), de menthe (lin. B: $m i-t a$ ), de pouliot (lin. B: $k a-r a-k o$ ), de carthame (lin. B: $k a-n a-k o$ ) ou de sésame (lin. B: sa-sa-ma); les haricots, les petits pois, les

\footnotetext{
${ }^{1}$ Cf. Athénée. Les Deipnosophistes. Texte établi et traduit par Desrousseaux, A.-M. Paris: Les Belles Lettres, 1956. Tome I, livres I et II/ I, 9a, p. 19-20.

${ }^{2}$ Cf. Athénée, op. cit., I, 9b-c, p. 21.

${ }^{3}$ Cf. Athénée, op. cit., I, 9d-e, p. 21.

${ }^{4}$ Cf. Vickery, K. F. Food in early Greece. Illinois Studies in the Social Sciences. Urbana, vol. XX, n. 3, 1936.

${ }^{5}$ Cf. Bruns, G. Küchenwesen in mykenischen Zeit. In: Vandenhoeck \& Ruprecht, 1970. Band II, Kapitel Q, p. 1-31.

${ }^{6}$ Cf. Dalby, A. The Gardens of Alcinous. In: in Greece. London: Routledge, 1996, p. 33-54. Archaeologia homerica. Göttingen: . Siren feasts. A history of food and gastronomy
} 
lentilles et la vesce, mais encore - et, selon K. F. Vickery, d'après des noms pré-grecs -

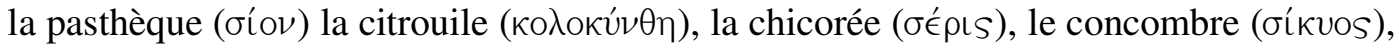

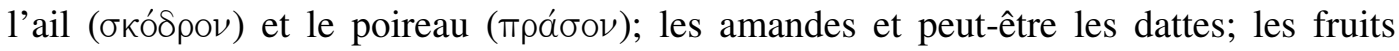
suivants: le coing (lin. B: ku-do-ni-ja), la poire, la figue, la prune, la grenade (et peutêtre la pomme); l'olive (lin. B: $e-r a-w a$ ) et l'huile d'olive (lin. B: e-ra-wo); le fromage (lin. B: tu-ro); le miel (déjà produit par l'apiculture); le vin (et peut-être la bière); les animaux domestiques suivants: le mouton, le porc, la chèvre et le bœuf, mais encore l'oie et le canard; les animaux sauvages comme gibier possible: le sanglier, le cerf, la chèvre, la perdrix et le lièvre; et enfin les poissons (parmi lesquels se distingue le thon), le poulpe, et des fruits de mer comme la seiche ou le calmar, les moules et les huîtres. ${ }^{7}$ Cette liste ne nous donne, pourtant, qu'une série d'éléments possibles dont 1'ordre ou un ensemble minimal peut rester encore un peu difficile à saisir. Pour essayer de suppléer à ce manque-là nous avons choisi une sorte de résumé descriptif apporté comme conclusion par K. F. Vickery à son livre sur la nourriture en Grèce à l'âge de bronze (résumé qui - en dépit de quelque ajout ou précision apportée par le déchiffrement du linéaire B - reste, dans son ensemble, valable):

Mutton and pork were the principal meats. With them, and probably forming a more important part of the diet, were eaten a considerable variety of leguminous vegetables. Coarse bread, probably unleavened, was made with wheat or barley meal. Some spices were available to season this food. Like later Mediterranean peoples, the pre-historic Aegean peoples probably prepared many salads with green vegetables, vinegar, and olive oil. They must also have had pickled olives and, as delicacies, certain fruits and nuts, such as apples, pears, plums, pomegranates, and almonds. The more fortunate, we may suppose, ate sweet prepared honey. As drinks they probably had beer and wine. In addition to these usual foods, the meats were supplemented, and in some places largely supplanted by sea food. Beef was sometimes eaten, though more rarely than mutton and pork - perhaps chiefly in connection with religious festivals. Especially among the upper classes fowl and wild game varied the $\operatorname{diet}^{8}$

\footnotetext{
${ }^{7}$ Pour le montage de cette liste aproximative voir les informations rassemblées par K. F. Vickery, dans les chapitres V (Linguistic evidence), VI (Plant products), VII (Domestic animals), VIII (Sea Food) et IX (Hunting) de Food in early Greece (cf. Vickery, op. cit., p. 45-85); par Gerda Bruns dans le sous-chapitre 1c Küchenwesen in mykenisher Zeit: Lebensmittel und Getränke de Archaeologia homerica (cf. Bruns, op. cit., p. 8-11; p. 63-67); et par Andrew Dalby dans les sous-chapitres The neolithic diet, Experiment and change, The roots of gastronomy, Prehistoric vegetables, Flavours and colours et Paths and patterns of innovation du chapitre 2 (The gardens of Alcinous) de Siren feasts (cf. Dalby, op. cit., p. 42-54).

${ }^{8}$ Cf. Vickery, op. cit., p. 89.
} 
Mais même si, de manière approximative, on voulait projeter sur l'âge de bronze historique en Grèce les temps heroïques de l'Iliade et de l'Odyssée, ${ }^{9}$ il resterait un peu naïf, du point de vue méthodologique, de vouloir procéder à une évaluation (ou vérification) de la nourriture mangée dans l'Odyssée par rapport à ce que les données archéologiques (et les documents comptables en linéaire B) nous renseignent sur ces temps-là, car la construction (en large partie imaginaire) de l'Odyssée n'obéit en aucune façon à un principe de vraisemblance stricte et peut - même dans le monde humain qui y est représenté - opérer par suppression, amplification, choix et rangement voulus des éléments qui (non nécessairement inventés) serviront à former un petit tableau habituel ou scène typique comme celle du repas. Mais pour avoir une idée plus précise-quant à la nourriture - des éléments possibles (lors du temps assez large de sá composition monumentale) et du choix que le poète en fait, il vaut mieux partir de ce que le poème lui-même revèle connaître comme nourriture possible, même si elle n'est jamais montrée en train d'être mangée (ou seulement dans des circonstances exceptionnelles). On pourrait avoir ainsi de même une idée du répertoire des éléments possibles (mais pas exactement du menu précis d'un repas qui en est composé: choix, quantité et ordre de consommation) d'une nourriture qui - même si sa consommation n'est pas (ou qu'exceptionnellement) décrite - reste reconnaissable pour l'auditoire comme mangeable. ${ }^{10}$ En étant un répertoire un peu plus large (dont quelques éléments furent déjà mentionnés comme le fromage, le lait et le miel) que celui d'un repas dans le monde humain de l'Odyssée (composé toujours du pain d'orge ou de blé, de la viande grillée des animaux domestiques et du vin mélangé avec de l'eau), il laisse deviner que l'auditoire devrait déjà bien percevoir le choix que faisait le poète de l'Odyssée en excluant systématiquement quelques uns de ces éléments du menu traditionnel "painviande-vin" du repas de ses héros ou personnages mortels dans le monde humain. Ce sont alors d'abord des éléments internes au texte même de l'Odyssée - et non pas

\footnotetext{
${ }^{9}$ Il n'est d'ailleurs pas du tout notre intention ici de reprendre la difficile et déjà très discutée question de l'historicité (ou du rapport à l'histoire) des poèmes homériques et ni non plus - ce qui serait peut-être plus utile mais très coûteux - de faire une petite histoire critique de la question elle-même.

${ }^{10}$ Cf. G. Murray [The rise of the Greek epic. Oxford: 1934, p. 121, cité par Davies, M. Feasting and food in Homer. Realism and stylisation. Prometheus. Firenze, fasc. II, p. 99 (note 9), 1997]: The heroes of the "Iliad" consume only heroic food, consisting chiefly of "unspeakable flesh and sweet strong wine". They eat enormous slices of roast ox or sheep or boar, and that three times a day. They do not condescend to boiled meat, much less to fish, fowl or vegetable, milk or cheese... In the similes, however, there is quite a lot about fishing (...). There are similes taken from the catching of larks and pigeons (...). There is much about milk and cheese, and one mention of boiled pork. That is the poet's own work-a-day world, where people had at most two meals a day and meat was a scarcity. L'intuition de Gilbert Murray est bonne, mais son essai de définition de la nourriture humaine dans le monde du poète reste tout à fait invérifiable.
} 
extérieures comme des données archéologiques - qui constitueront un arrière-fonds d'un savoir plus large partagé par le poète et l'auditoire et qui permettra, par comparaison, une première perception de la spécificité du repas héroïque (ou mortel) traditionnel dans le monde humain de l'Odyssée. Si nous voulions sortir un peu de l'Odyssée (et de l'Iliade) pour élargir adéquatement le cadre d'un savoir partagé contemporain sur la nourriture, ce serait plutôt Hésiode (Théogonie et Travaux) qui, avec sa définition mythique de l'homme par le travail de la terre et par le sacrifice (qui fournissent respectivement le pain/vin et la viande pour les repas), aiderait a éclairer la logique des habitudes alimentaires des personnages humains de l'Odyssée. ${ }^{11}$

\section{Les fruits (et l'huile d'olive)}

Voyons donc, pour commencer, les fruits qui - comme on le sait bien n'apparaissent sur des tables et ne font jamais partie d'un repas dans l'Odyssée. On pourrait d'abord y exclure comme tout à fait improbables "le fruit du cornouiller" (карто́ comestible -, parce que là (ainsi que le gland et la faîne) il est désigné comme la nourriture des compagnons d'Ulysse transformés par Circé: "Ce que mangent toujours les cochons vautrés à terre" $(\text { Od. X, 243 })^{12}$, et les dattes dont n'est cité que le palmier,

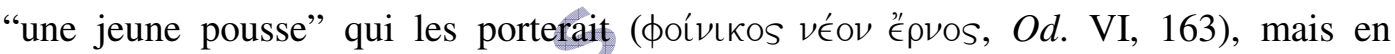
l'occurrence sûrement comme plante ornementale (de l'autel d'Apollon à Delos) à laquelle est comparée la belle et jeune Nausicaa (cf. Od. X, 160-168), car ses fruits ne mûrissent pas en Grèce. ${ }^{13}$ Ensuite il y a des mentions génériques et colectives d'arbres fruitiers qui ne sont pas nommés individuellement: celle plus vague du prétendant Eurymaque qui - pour critiquer la paresse d'Ulysse-mendiant - lui offre ce travail agricole: “Couper de l'épine et planter de grands arbres ( $\delta \epsilon ́ \nu \delta \rho \in \alpha \mu \alpha \kappa \rho \alpha ́)$ (Od. XVIII,

\footnotetext{
${ }^{11}$ C'est ce qui d'ailleurs a déjà été démontré (encore que non pas toujours directement pour ce qui est de la nourriture) par l'article de Pierre Vidal-Naquet (Valeurs mythiques et religieuses de la terre et du sacrifice dans 1' “Odyssée”. In:___ Le chasseur noir. Paris: Maspero, 1981, p. 39-68). Sur un savoir partagé avec le destinataire et intérieur au texte même de l'œuvre (comme instrument de lecture), voir les remarques méthodologiques de François Hartog sur les Scythes d'Hérodote dans l'introduction de la première partie du Miroir d'Hérodote (Hartog, F. Les Scythes d'Hérodote: le miroir scythe. In: Le miroir d'Hérodote. Essai sur la représentation de l'autre. Paris: Gallimard, 2001, p. 5767).

${ }^{12}$ Cf. Dalby, op. cit., p. 80: The cornel [i.e. a minor fruit], "kránon”, was edible but probably best known as having been food for Circe's pigs in the "Odyssey" (10. 242) (...).

${ }^{13}$ Cf. Dalby, op. cit., p. 82: Date palms had been planted in Crete in the bronze age; Odysseus' narrative ("Odyssey" 6.123) speaks of a date palm growing at Apollo's shrine on Delos, but these would have been ornamental trees, for dates not ripen in Crete or Greece. Voir aussi Richter, W. Archaeologia homerica. Göttingen: Vandenhoeck \& Ruprecht, 1968. Band II, Kapitel H, p. 144.
} 
359); et celle plus explicite dans l'image d'abondance du royaume du roi juste auquel Ulysse-mendiant compare Pénélope: “(...) La terre noire produit/ les orges et les blés,

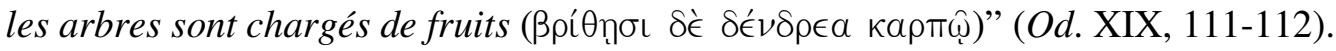

Lorsque les arbres fruitiers sont nommés, ce qui a lieu trois fois dans tout le poème, ils forment un même groupe de cinq (ou quatre, si l'on enlève le grenadier, mais respectant en quelque manière l'ordre de nomination), dont le caractère formulaire fut bien perçu par Will Richter qui dit que c'est comme si ce groupe-là exprimait ou épuisait le concept même de "fruit" dans l'Odyssée: poiriers, pommiers, grenadiers, figuiers et oliviers (auxquels peut aussi s'ajouter la vigne). ${ }^{14}$ La première occurrence, celle au jardin/ verger d'Alcinoos, a quelque chose de phantastique non pas à cause de fruits eux-mêmes mais plutôt du rythme acceleré de leur croissance quii, stimulée par le bon climat constant, ne respecte pas les saisons et fait penser à une sorte d'âge d'or: ${ }^{15}$

Là de grands arbres ont poussé avec richesse,

des poiriers, des pommiers aux fruits brillants, des grenadiers,

des figuiers doux, des oliviers en pleine force.

Ni l'été ni l'hiver les fruits ne font défaut, toute l'année les arbres donnent, et sans relâche, un doux Zéphyr fait bourgeonner les uns, mûrir les autres. ${ }^{16}$

La deuxième occrrence, celle à la scène de Tantale au chant XI, qui répète tels quels les vers 115-116 du chant VII, a aussi - en tant que scène cruelle de châtiment et par son contexte imaginaire bien marqué: l'Hadès - quelque chose de phantastique, mais elle laisse deviner que ces fuits (jamais explicitement mangés) étaient bien figurés comme une nourriture tout à fait désirable: ${ }^{17}$

Au dessus de sa tête, de hauts arbres offraient leurs fruits, des poiriers, des pommiers aux fruits brillants, des grenadiers, des figuiers doux, des oliviers en pleine force:

${ }^{14}$ Cf. Richter, op. cit., p. 140: (...) In jedem Fall weckt sie den Eindruck, als solle sie für den Begriff 'Obst' schlechtin stehen, wenn nicht überhaupt ihn erschöpfen.

${ }^{15}$ Cf. Vidal-Naquet, op. cit., p. 61: (...) Il ne s'agit pas que d'un verger, c'est bien un îlot de l'âge d'or au cœur de la Phéacie.

${ }^{16}$ Od. VII, 114-119. Cf. Homère. L' "Odyssée”. Traduction et notes de Philippe Jaccottet. Paris: Maspero, 1982. Le texte grec adopté est celui établi par Von der Mühll, P. Homeri Odyssea. Leipzig: Teubner, 1983.

${ }^{17}$ Athénée l'avait bien perçu lorsque - après avoir rappelé que Homère les montre s'occupant également des arbres fruitiers: "Poire vieillit sur poire", dit-il quelque part (Od. 7, 114), "et figue sur figue”. - il note: Mais à plus haut que l'époque de Troie remonte l'usage des fruits. Ainsi Tantale, même après sa mort, n'en perd pas la convoitise, puisque le dieu qui le punit agite devant lui de ces fruits (...) et l'empêche ensuite d'en goûter chaque fois qu'il en arrive à l'espoir proche (cf. Athénée, op. cit., I 25a-b, p. 57-58). 
à chaque fois que le vielliard essayait d'y porter la main, le vent les rejetait vers les nuages sombres. ${ }^{18}$

La troisième occurrence, pourtant, même si le grenadier n'est pas mentionné (et la vigne est ajoutée), n'a pas lieu dans un contexte extraordinaire ou phantastique, car c'est bien le verger de Laërte, objet du travail humain et soumis aux conditions naturelles, (ce qui devient clair quand Ulysse parle de ceps de vigne: "Il y a là des grappes de toutes les sortes,/ quand les saisons de Zeus du haut du ciel les alourdissent". Od. XXIV, 343-344), et qu'on aurait du mal à imaginer si les fruits n'étaient pas produits pour être mangés (ou convertis en une façon quelconque de nourriture). Lorsque, encore non identifié, Ulysse s'adresse à son père, il fait une première description de son verger:

On voit, vieillard, que tu connais les travaux du jardin: tout ici montre de grands soins, et il n'est rien, ni plante, ni figuier, ni olivier, ni vigne,

ni poirier, ni légume qui paraisse négligé. ${ }^{19}$

Mais c'est quand il s'identifie qu'Ulysse va non seulement nommer (alors dans l'ordre traditionnel, même si le grenadier est absent et l'olivier est remplacé par la vigne), mais aussi dénombrer les arbres fruitiers que Laërte lui avait donnés lorsqu'il était encore enfant (ce qui signale peut-être une sorte d'initiation à l'art de la plantation et du soin de ce genre d'arbres):

Tû me donnas alors treize poiriers et dix pommiers et quarante figuiers; de même, tu me désignas cinquante rangs de ceps dont chacun était vendangeable (...). ${ }^{20}$

Cette double description du verger de Laërte par Ulysse confirme donc celle du verger d'Alcinoos et indique nettement dans l'Odyssée la pratique de la plantation et du maintien des arbres fruitiers, ce qui d'ailleurs n'est pas attesté par les données archéologiques de l'âge du bronze. ${ }^{21}$ On pourrait peut-être ajouter que le premier fruit comestible dont il y a quelque notice en Grèce pré-historique c'est une sorte de poire

\footnotetext{
${ }^{18}$ Od. XI, 588-592.

${ }^{19}$ Od. XXIV, 244-247.

${ }^{20}$ Od. XXIV, 340-342.

${ }^{21}$ Cf. Dalby, op. cit., p. 77: The "Odyssey" tells us, then, of the planting of orchards, a practice for which there is no prehistoric archeological evidence in Greece.
} 


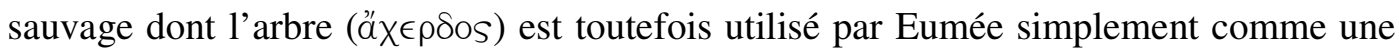
espèce de haie de protection $(O d . \mathrm{XIV}, 10){ }^{22}$

Andrew Dalby, après avoir cité la description du verger d'Alcinoos (Od. VII, 112-121), essaie d'imaginer - à partir de sources grecques plus tardives - l'usage possible à la table de ces cinq fruits (et, en plus, du raisin), en distinguant deux types: les fruits juteux comme un amuse-gueule ou un hors d'œuvre, et les fruits sucrés (ainsi que les noix) comme une sorte d'accompagnement du vin servi après le repas (selon le modèle du sympósion). ${ }^{23}$ D'après cette typologie binaire, Dalby considère alors les

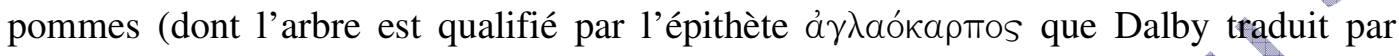
"shiny-fruited") et les olives comme des hors d'œuvre, tandis qu'il considère les poires, les grenades et les figues (dont l'arbre, бUкé $\eta$, féminin en grec, est qualifié de $\gamma \lambda \nu \kappa \in \rho \eta ́$ "douce") et les raisins (aussi séchés) comme de "deuxièmes plats" ou accompagnements du vin. $^{24}$ Or, le grand problème, pour nous interprètes de l'Odyssée, de cette classification de Dalby est - outre le fait primaire qu'elle est entièrement invérifiable dans l'Odyssée, car ces fruits n'y sont mangés nulle part - sa formulation anachronique à partir du modèle du sympósion ("banquet" au sens classique) où, comme on le sait bien, le repas ne constitue qu'une sorte de préparation, après laquelle seulement on peut commencer à boire du vin et après laquelle aussi on ne mangera plus une nourriture forte comme la viande, tandis que dans les repas homériques le vin accompagne la nourriture principale (qui est d'ailleurs la seule: le pain et la viande) qui peut, à son tour, continuer à être mangée, (et n'est donc pas enlevée des tables) après un premier moment de satisfaction. Dans ce modèle plus simple (et à la fois comportant le mélange de nourriture et boisson) on voit mal quelle serait la place à part de ces cinq fruits, car il n'y a là ni d'amuse-gueule ni de dessert ou deuxième plat léger pour accompagner le vin.

D'ailleurs, de même que dans l'Odyssée le vin est la seule utilisation visible des raisins (jamais mangés comme deuxième plat), de même les olives (jamais mangées

\footnotetext{
${ }^{22}$ Cf. Dalby, op. cit., p. 39: The first fruit known to have been used by humans in Greece was a species of pear. "Pyrus spinosa" ist the wild pear of south-eastern Europe: its fruits can be eaten fresh but are better cooked or dried. This thorny wild pear was later planted nor for its fruits so much as for its use in a defensive hedge. It is the "akherdos" with which Eumaeus' farm is fortified in the "Odyssey" (14.10).

${ }^{23}$ Cf. Dalby, op. cit., p. 77: Later sources fill in the gap for us: juicy fruits might be appetisers; sweet fruits, dried fruits and nuts were served on the "second table" to be chewed alongside wine, and likewise accompanied wine at "symposia".

${ }^{24}$ Cf. Dalby, op. cit., p. 77-80.
} 
comme hors d'œuvre, ${ }^{25}$ même si la scène de Tantale suggère qu'elles pourraient aussi

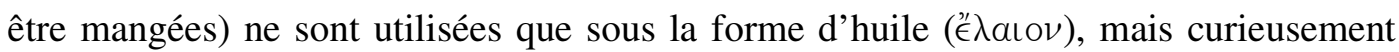
jamais dans la cuisine ou à la table et seulement pour oindre la peau après les bains, ainsi que nous le montrent les scènes typiques de bain dans l'Odyssée avec un vers formulaire qui, avec de legères variations, dit toujours de manière succinte l'action de laver et de frotter d'huile (cf., par exemple, Od. III, 466; IV, 49; VI, 96 et 227; VIII, 454, X, 364; XVII, 88; XIX, 154). Au chant VI la fonction de l'huile d'olive est rendue plus évidente, lorsqu'Areté prépare les provisions pour le petit voyage de Nausicaa: le poète alors ne mentionne l'huile (en explicitant bien sa fonction) qu'après avoir fini de décrire ce qu'elles amèneront comme nourriture pour leur pic-nic:

(...)

sa mère, en un panier, mit des vivres en abondance, de toute espèce, et des douceurs; elle versa du vin dans une outre de chèvre; et la jeune fille monta. Sa mère lui donna une fiole d'or pleine d'huile pour qu'elle s'en oignît après le bain avec ses femmes. ${ }^{26}$

\section{Les légumes}

Essayons maintenant de voir ce qu'il y a - ou même s'il y en a - de mentions de (ou sur des) légumes dans l'Odyssée, car aux scènes de repas (ou sur les tables) on sait bien qu'ils ne sont jamais présents. ${ }^{27}$ L'Iliade présente, pourtant, une seule occurrence d'un légume au point d'être mangé: celle déjà citée de l'oignon (крó $\mu v o v)$ "pour

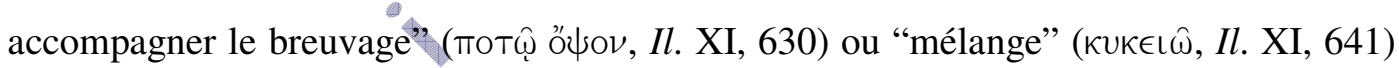
de vin de Pramnos, fromage de chèvre râpé et farine blanche, qui est préparé par Hécamède comme un fortifiant pour Nestor et Machaon blessé qui viennent de rentrer

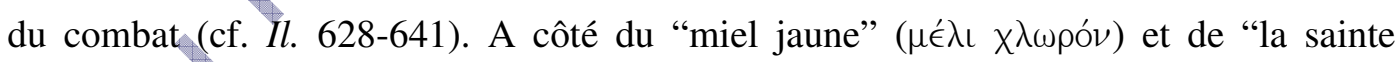

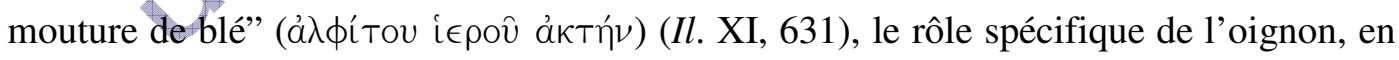

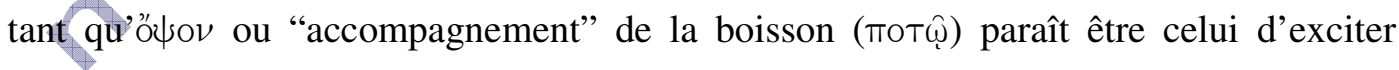
l'envie de boire (ou de renouveler la soif) comme un amuse-gueule acide. L'autre et

\footnotetext{
${ }^{25}$ Cf. Dalby, op. cit., p. 78: The olive, "elaia”, was more important as a source of oil than as a table fruit, yet olives were a popular appetiser, wrinkled or underripe or fully ripe, drypepés (...). Pour l'utilisation des raisins voir aussi Dalby, op. cit., p. 80: The main economic importance of the grape was of course in wine-making (...), but the fruit itself belonged among desserts, for which purpose fresh grapes were preserved in wine. (...) Grapes were also, of course, dried, and as such served both for dessert and as flavouring in cuisine.

${ }^{26} \mathrm{Od}$. VI, 76-78.

${ }^{27}$ La phrase générique de Will Richter s'applique parfaitement à l'Odyssée: In keiner Tafelszene des Epos spielen Gemüse eine Rolle, gleich als hätte man sie nicht gekannt (cf. Richter, op. cit., p. 124).
} 
double occurrence de légumes dans l'Iliade, celle de fèves noires

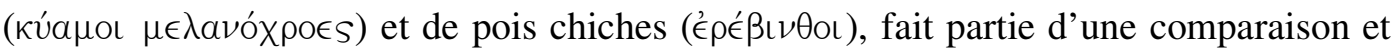
ne les présente pas sur la table mais en train d'être vannés comme le blé, ce qui indique - pour les auditeurs mais pas pour les personnages - une préparation première pour une possible conversion en nourriture, car c'est le poète qui raconte alors comment Hélénos essaie en vain de blesser Ménélas avec une flèche: "Ainsi, de la large pelle à vanner, sur une aire immense, sautent fèves noires ou pois chiches, dociles au vent sonore et à l'élan donné par le vanneur; ainsi, de la cuirasse du glorieux Ménélas, la flèche amère rejaillit et se perd au loin" (Il. XI, 588-92). ${ }^{28}$

Il y a cependant dans l'Iliade une autre plante cultivée dont l'utilisation n'est pas définie (ni non plus déductible) et reste donc entièrement ouverte à des conjectures: le pavot $(\mu \eta \hat{\kappa} \kappa \omega \nu)$. Il apparaît, lui aussi, à l'intérieur d'une comparaison dans le récit du poète qui paraît ainsi en préssuposer une reconnaissance possible (même si elle n'est pas explicitée) de la part de l'auditoire. Voici comment le poète raconte la réaction physique de Gorgythion atteint à la poitrine par une flèche létale de Teucros: "Tel un pavot, dans un jardin, penche la tête de coté, sous le poid de son fruit et les pluies printanières, tel il penche son front par le casque alourdi" (Il. VIII, 306-308). Or, une utilisation médicinale du pavot - analogue à celle de l'opium - est imaginée par Will Richter à partir de la première description des effets de la drogue ( $\phi a ́ p \mu \alpha \kappa o v)$ mélangée par Helène au vin qu'elle servira à son mari, à Télémaque et à Pisistrate, qui alors pleuraient, car c'est une puissante drogue sédative "qui efface la douleur et la colère"

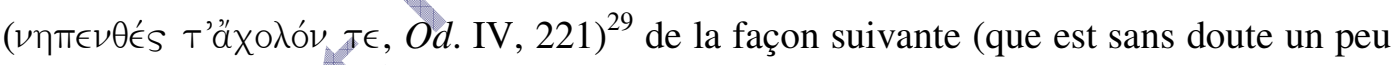
magique):

quiconque en avalait, mélangée au vin du cratère, de tout le jour ne répandait plus une larme, êut-il même perdu ses parents, son père et sa mère, êut-il vu devant lui, et de ses propres yeux, son frère ou son bien-aimé fils succomber sous l'épée! ${ }^{30}$

\footnotetext{
${ }^{28}$ Cf. Homère. Iliade. Traduction de Paul Mazon, préface de Pierre Vidal-Naquet. Paris: Gallimard, 1995. Le texte grec adopté est celui de Monro, D. B.; Allen, T. W. (org.): Homeri opera. Oxford: University Press, 1988-1989. Tomi I-II [1ed. 1902]. Voir le commentaire de Richter, op. cit., p. 124: Die eingize

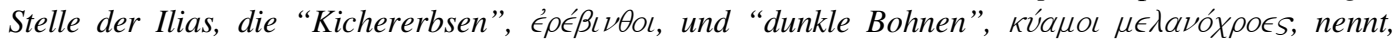
verrät zugleich, dass man sie genau wie das Getreide auf der Tenne ausgedroschen hat; sie wuchsen also wohl auch wie dieses auf dem Acker.

${ }^{29}$ Cf. Richter, op. cit., p. 126: Die einzige Pflanze von offizieller Bedeutung, die sicher in Gärten augebaut wurde, ist der Mohn, der zu einem Analgetikum verwendet wurde (voir aussi la note 960 avec la citation de Il. VIII, 306 et de Od. IV, 221).

${ }^{30}$ Od. IV, 222-226.
} 
Le problème le plus grand de l'association de ces effets au pavot est qu'il n'est pas du tout mentionné quand le poète raconte plutôt de manière générique la provenace égyptienne de cette drogue: ${ }^{31}$

Hélène disposait ainsi de main charme (фápнaka) rusé que lui avait fourni Polydamna, femme de Thon, en Égypte où la terre aux blés produit en abondance toute espèce de simples ( $\phi a ́ \rho \mu a \kappa a)$, salutaires ou funestes, (...). ${ }^{32}$

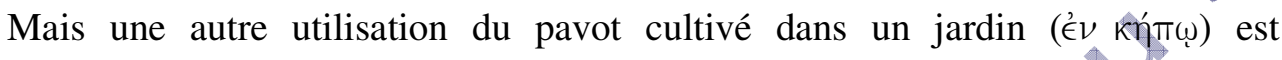
imaginable - encore qu'invérifiable dans l'Iliade - à partir des attestations depuis Alcman (19) et Thucydide (4.26.8) réunies par Andrew Dalby qui parle d'une épice faite de pépins de pavot pour assaisonner les pains ou des plats cuits. ${ }^{33}$ Phyllis Pray Bobber suggère encore un autre mode d'usage du pavot (lui aussi invérifiable dans l'Iliade) à partir des occurrences du mot sur les tablettes en linéaire B: comme huile, dont la fonction serait aussi de donner du goût ou celle de parfumer. ${ }^{34}$

Dans l'Odyssée, pourtant, tout ce qu'il y en a ce sont deux occurrences d'un seul

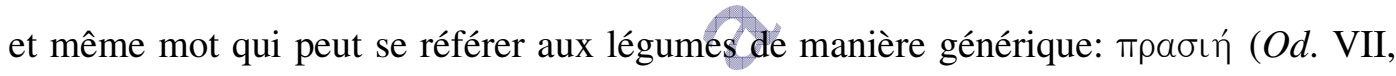
127 au nom. pl., et XXIV, 247), terme de horticulture qu' on pourrait traduire par "platebande de légumes", mais éventuellement aussi " plate-bande de fleurs", ou simplement “plate-bande", vu que le genre de plante cultivée n'y serait pas explicitement exprimé, encore que l'étymologie (ou une simple dérivation) renvoie sûrement à $\pi \rho a ́ \sigma o \nu$,

\footnotetext{
${ }^{31}$ Voir aussi le commentaire plutôt historique de Stephanie West (Books I-IV. In: Heubeck, A.; West, S.; Hainsworth, J. B. A commentary on Homer's "Odyssey”. Oxford: Clarendon Press, 1988. Vol. I, p. 49245/ p. 206-207): It has often been suggested that the description of Helen's wonderful drug is based on opium, which is not mentioned in Greek literature before the fourth century and cannot have been widely known in Greek lands when the "Odyssey" was composed; henbane ("Hyoscyanus niger") has also been proposed. But there is no reason to think that it was costumary in antiquity, either in Egypt or anywhere else, to add either drug to wine; nor indeed is it quite certain that opium was known in Egypt at this period.

${ }^{32}$ Od. IV, 227-230.

${ }^{33}$ Cf. Dalby, op. cit. p. 86: Poppy, “mékon”, the seeds used as a garnish for loaves and as a flavouring for cooked dishes (the leaves were also eaten); et aussi la note 189 relative à ce passage, p. 237: Alcman 19 [Athenaeus 111a]; cf. Philostratus, "Gymnasticon" 44; Pliny, "Natural History" 9.12.4; Epicharmus 159-61 [Epitome 70f-71a]. (...) Thucydides, "Histories" 4.26 .8 corroborates Alcman on the use of poppy seeds as food at Sparta.

${ }^{34}$ Cf. Bober, P. P. Art, culture and cuisine. Ancient and medieval gastronomy. Chicago: The University of Chicago Press, 1999, p. 88: The range of spices and aromatics is impressive in Linear B archives, as are multiple attributes for differentiating oils by provenance and age (...) and flavours (from seeds and extracts of roses, sage, mint, and various herbs). (...) Perhaps the poppies that figure in the lists served as another source for oil just as the seed had been exploited from Neolithic times in other parts of Europe.
} 
"poireau", Allium porrium. ${ }^{35}$ Voyons la première occurrence, celle au jardin/verger d'Alcinoos, juste après la description des arbres fruitiers et des ceps de vigne:

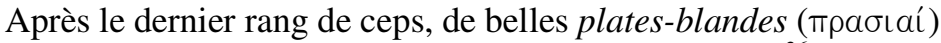
donnent toutes les plantes et verdoient en toute saison. ${ }^{36}$

Ce passage nous indique - outre le fait magique, comme dans le cas des arbres

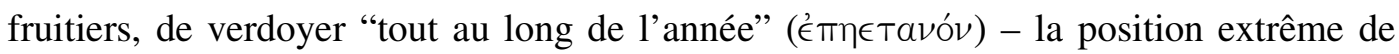

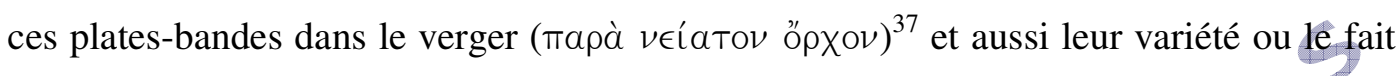

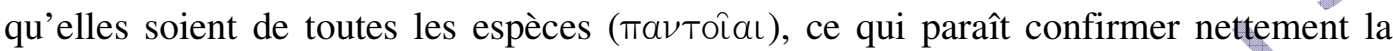
généralité du sens ou sa non restriction aux légumes, mais si l'on pense à l'ensemble du contexte formé seulement des arbres fruitiers (dont les fruits, comme nous l'avons dit, sont censés donner de la nourriture) et où aucune plante ornementale est citée, on serait plutôt enclin à penser à des plantes-bandes de légumes que de fleurs. ${ }^{38}$

La deuxième occurrence du mot $\pi \rho a \sigma ı n ́$, celle au verger de Laërte, permet (comme dans le cas des arbres fruitiers) d'attester que ces plantes croissaient aussi normalement selon les saisons dans des vergers qui n'ont rien de magique, mais encore une fois le contexte suggère fort bien qu'il s'agit plutôt d'un jardin potager (ou verger) que d'un jardin avec des plantes ornementales, ce qui transparaît cette fois dans la traduction (d'ailleurs déjà citée) de Philippe Jaccottet pour ces paroles d'Ulysse à son père:

\footnotetext{
${ }^{35}$ Le Dictionnaire étymologique de langue grecque de Pierre Chantraine (Dictionnaire étymologique de

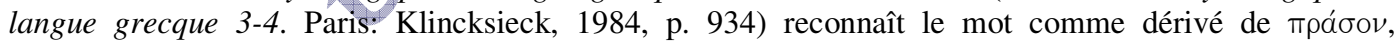
"poireau", mais lui donne un sens générique: "Plate-bande d'un jardin" (carré de légumes ou de fleurs, Od. Thphr., LXX, etc.), ce que suggère aussi le Lexikon des frühgriechischen Epos [Snell, B. (begründet von); Meier-Brüger, M. (Redaktor). Lexicon des frühgriechischen Epos. Göttingen: Vandenhoeck \& Ruprecht, 2004. Band III, p. 1506]: Spez. Lauchbeet? od. bereits allg. "Grün(zeug)beet” (vgl. später

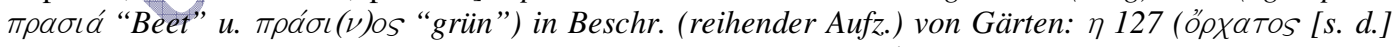

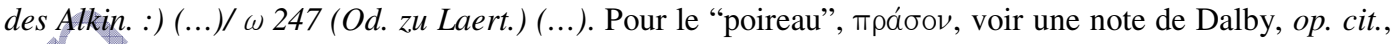
p. 235: Surely the garden leek, "Allium porrum” (...) though it has been argued that the leek grown in Egypt in the second millenium BC was "Allium kurrat", still known in the Near East. Le texte de A. Dalby dit: Leek, "práson", a plant that apparently defined horticulture in archaic Greece to the extent of having provided the Homeric terme for a garden bed, "prasía” ("Odyssey” 7.127, 24.247) (...). ${ }^{36}$ Od. VII, 127-128.

37 Le Lexicon homericum de H. Ebeling cite l'Etymologicum Magnum (686, 48)

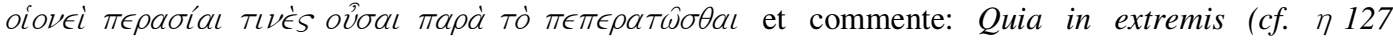

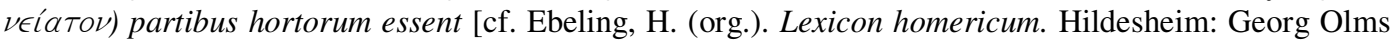
Verlag, 1987. Vol. II, p. 221]. Mais que la forme de ces plates-bandes soit carrée ou rectangulaire n'est

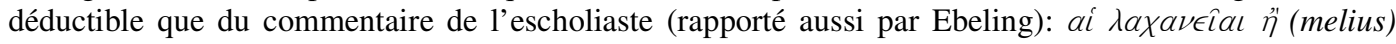

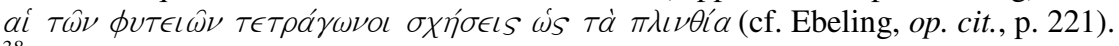

${ }^{38}$ Voir aussi ce que dit Athénée, op. cit., I 24f, p. 57: On servait aussi aux héros des légumes dans les repas. Le fait qu'ils connaissent la culture des légumes est rendu évident par les planches bien soignées "de la dernière rangée" ("Odyssée” 7, 127).
} 
On voit, vieillard, que tu connais les travaux du jardin: tout ici montre de grands soins, et il n'est rien, ni plante, ni figuier, ni olivier, ni vigne

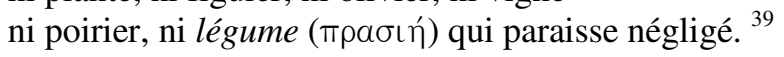

On pourrait enfin ajouter - d'après une indication du scholiaste A de l'Iliade (XVI, 747) - une allusion possible à des plantations de légumes (mais pas seulement ou spécifiquement d'eux) dans la mention - lors de l'épisode du chien Argos qui s'y

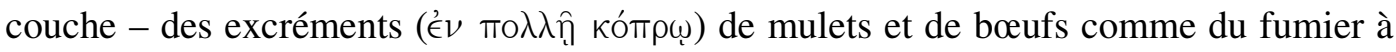

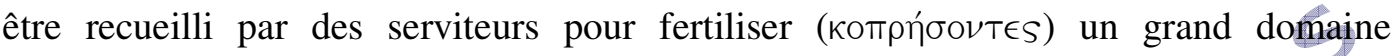

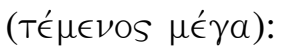

Mais maintenant il gisait là sans soins, le maître absent, sur du fumier de bœuf et de mulet qu'on entassait devant la porte, afin que les valets d'Ulysse eussent toujours de quoi fumer les grands domaines. ${ }^{40}$

C'est lorsqu'il commente la connaissance des huîtres par Patrocle, même si les héros de l'Iliade ne mangent pas de poisson, que le scholiaste A suggère - en parlant du poète - la comparaison suivante: Et il n'introduit pas non plus (des héros) qui mangent (littér. se servent) des légumes. Mais néanmojns il dit "les serviteurs d'Ulysse sur le point du fumer un grand domaine" $(\rho$ 299)

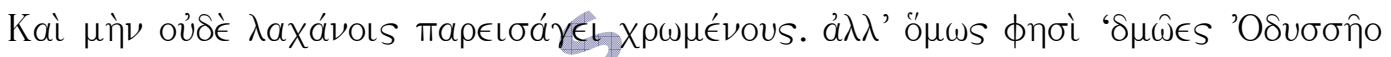

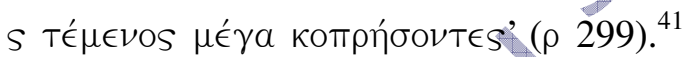

\section{Les oies de Pénélope}

Si nous passons maintenant aux animaux domestiques qui - en plus de moutons, porcs, chèvres et bœufs - pourraient être envisagés comme mangeables (même si aucune scène de repas ne l'atteste dans l'Odyssée), ce ne serait évidemment pas aux

\footnotetext{
${ }^{39}$ Od. XXIV, 244-247.

${ }^{40}$ Od. XVII, 296-299.

${ }^{41}$ Erbse, H. (org.). Scholia Graeca in Homeri "Iliadem" (Scholia vetera). Berlin: Walter de Gruyter, 1975. Vol. IV, p. 295. La paraphrase de ce commentaire du scholiaste A par Martin Schmidt vaudrait

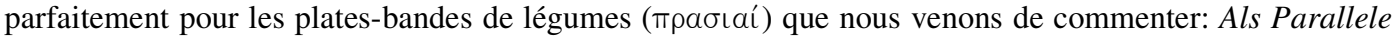
wird angeführt, dass bei Homer auch nie vom Essen von Gemüse die Rede ist, obwohl doch zweifellos solches angebaut worden ist. Dans une note assez concise, Martin Schmidt (Die Erklärungen zum Weltbild Homers und zur Kultur der Heroenzeit in den b-T Scholien zur Ilias. Zetemata. München, Heft LXII, p. 183, 1976) ajoute pourtant: Dafür wird als Beleg des Vers $\rho 299$ angeführt, in dem vom Düngen eines Feldes die Rede ist. Es ist nicht einleuchtend, warum dies ein Beweis für den Anbau von Gemüse sein soll (...).
} 
chiens (ou aux chevaux) qu'on pourrait penser ${ }^{42}$, mais aux vingt oies qui mangent du blé aussi bien dans le fameux rêve de Pénélope (Od. XIX, 536-537) que dans son palais réel lorsqu'elle se réveille (Od. XIX, 551-552). Il est vrai, pourtant, qu'il y a une autre occurrence d'une oie, celle qui (à la sortie de Télémaque du palais de Ménélas) est prise par un aigle dans un événement qui est perçu comme un signe et interprété par Hélène comme ayant rapport au retour et à la vegeance d'Ulysse (cf. Od. XV, 160-178). Cette oie $(\chi \eta \hat{\nu} \alpha)$ particulière et isolée est d'abord décrite (Od. XV, 161-162) comme

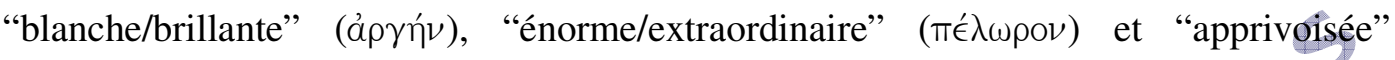

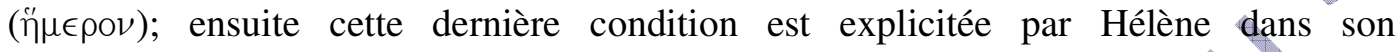
interprétation du signe, lorsqu'elle qualifie l'oie (Od. XV, 174) comme "élevée à la

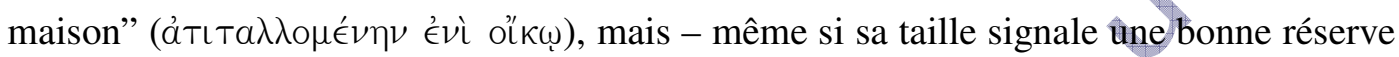
possible de viande - rien de précis ne nous indique quelle serait la fonction de son élévage.

Revenons maintenant aux vingt oies qui mangent du blé au début du rêve annonciateur de Pénélope (où elles seront tuées par un aigle) et aussi - comme pour marquer la fin du rêve et encadrer le récit de Pénélope dans une composition annulaire -

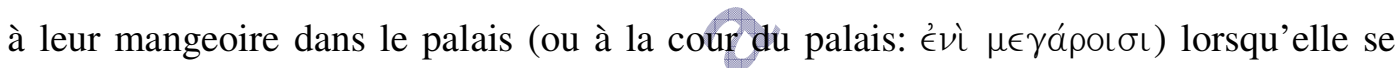
réveille:

Mais voyons! Écoute ce songe, et explique-le-moi : je vois vingt oies dans la maison manger du grain au sortir de l'étang ${ }^{43}$, et je m'attendris de les voir, (...). A ces mots, le sommeil doux comme miel m'abandonna; cherchant des yeux, je vis les oies dans le palais piquant le grain dans la mangeoire comme à l'ordinaire... ${ }^{44}$

A l'intérieur même du rêve se pose déjà la question du sens de l'évenement extraordinaire (prodige) qui est la mort violente de ces vingt oies par un grand aigle qui vient de la montagne et, après les avoir tuées en cassant leurs cous, regagne l'éther brillant (cf. Od. XIX, 538-540). Pour l'aigle qui revient (et parle pour rassurer Pénélope qui pleure) les oies sont les prétendants, et l'aigle lui-même, son mari revenu pour les

\footnotetext{
${ }^{42}$ Cf. Dalby, op. cit., p. 61: Both [i. e. pig and dog] were eaten in classical Greece, but dog, "kyon”, was not a food of which one boasted. It is never listed in the comedy menus or in the gastronomic poetry of the fourth century. Horse, "hippos", was a very uncommon choice as a food among Greeks, though its dietary qualities are assessed in Regimen (46).

${ }^{43}$ Dans une note Philippe Jaccottet ajoute: D'autres comprennent plutôt: "du grain trempé d'eau" (Homère, op. cit., p. 321).

${ }^{44}$ Od. XIX, 535-537; 551-553.
} 
tuer tous (cf. Od. I, 544-550). Pour cette interprétation, la seule à être explicitée, le fait

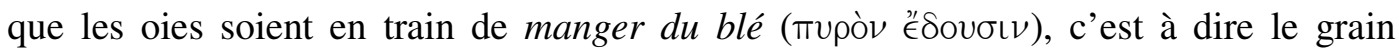
d'une céréale (comme celle qui fournit le pain aux hommes), manger qui d'ailleurs est l'unique activité qui les caractérise, ce fait peut sans doute être mis en rapport - comme le suggère Joseph Russo - avec le fait que l'activité qui caractérise en premier lieu les prétendants (qui consomment sans arrêt le patrimoine d'Ulysse dans leurs banquets) est celle de manger. ${ }^{45}$ Mais, dans le rêve, avant l'interprétation de l'aigle, la réaction fort endeuillée de Pénélope à la mort prodigieuse de ces vingt oies (Je pleurai et je criai (...)/ geignant piteusement sur le massacre de mes oies, Od. XIX, 541 et 543) peut laisser supposer une autre et bien différente interprétation du prodige, dans laquelle les oies auraient un sens symbolique positif mais ne s'identifieraient pas forcément aux prétendants (comme s'il aurait pu y avoir là un quelconque désir refoulé de Pénélope ${ }^{46}$ ). Dans un bref et éclairant article ("Odyssey 19.535-50: on the interpretation of dreams and signs in Homer"), Louise Pratt propose de lire d'abord avec plus d'attention le

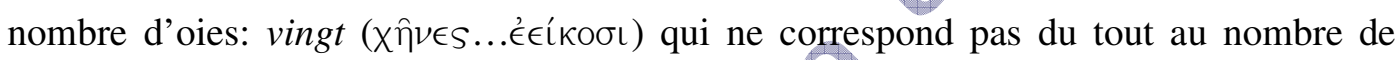
prétendants, mais plutôt à celui - bien connu - des années d'absence d'Ulysse et aussi d'une attente loyale de la part de Pénélope. ${ }^{47}$ Ensuite - en reprenant avec précaution l'association des oies (et aussi du nom de Pénélope) avec la fidelité conjugale, telle que l'a proposée P. Kretschmer à partir de la tradition chinoise - elle rappelle que dans la littérature grecque les oies (surtout celles domestiques) sont associées à une prudente surveillance de la maison, ${ }^{48}$ et propose donc que dans ce rêve les (vingt) oies représenteraient une fidèle surveillance de la maison par Pénélope (pendant vingt ans).

\footnotetext{
${ }^{45}$ Cf. Joseph Russo (Books XVII-XX. In: Russo, J.; Fernández-Galiano, M.; Heubeck, A. A commentary on Homer's “Odyssey”. Oxford: Clarendon Press, 1992. Vol. III, p. 1-127/ p. 102): The symbolism of this dream is reinforced by the fact that the single activity that caracterizes the geese is eating (535), which is the most conspicuous activity of the suitors.

${ }^{46}$ Cf. Devereux, G. Penelope's character. Psychoanalytic Quarterly. New York, vol. XXVI, p. 378-86, 1957. Et Russo, J. Interview an after-math. Dream, fantasy, and intuition in "Odyssey" 19 and 20. American Journal of Philology. Baltimore, n. 103 , p. 4-18, 1982.

${ }^{47}$ Cf. Pratt, L. "Odyssey" 19.535-50: on the interpretation of dreams and signs in Homer. Classical Philology. Chicago, n. LXXXIX, p. 150, 1994: Numbers tend to have significance in bird-signs. There are not twenty suitors. But twenty is a significant number in the "Odyssey", for it is said repeatedly that this is the twentieth year that Odysseus has been away.

${ }^{48}$ Cf. Pratt, op. cit., p. 151: Citing ancient Chinese folklore and litterature, Kretschmer argues that the etymological and mythological connection between Penelope and the "penelops", a kind of duck or goose (the two are not perfectly distinguished), should be explained by their shared reputations for marital fidelity. (...) Better attested in Greek literature is the association of geese, particularly domestic geese, with the prudent guardianship of the house. Aristotle, for example, gives the goose the character of a

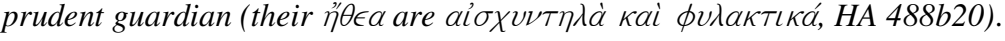


De toute façon, ce qui concerne vraiment notre sujet (les animaux domestiques comme source possible de nourriture) ce n'est pas le symbolisme onirocritique des oies (qui, comme nous venons de le voir, reste ouvert à des interprétations différentes), mais le fait attesté comme réel dans l'Odyssée que Pénélope possède vingt oies dans le palais et qu'elles sont vues "piquant le grain dans la mangeoire comme à l'ordinaire..." (Od. XIX, 553). On pourrait alors se poser la question: pourquoi sont-elles élevées et (à ce qu'il paraît) spécialement nourries au palais? K. F. Vickery, par exemple, propose - à partir du caractère ornemental et sacré des oies dans l'Égypte ancienne (même s'il suggère que l'usage des oies et de leurs œufs comme nourriture serait indiqué en Grèce pré-historique par des restes trouvés dans une tombe à Thèbes) - que dans l'Odyssée les oies, par association avec le rêve augural de Pénélope, seraient considérées comme sacrées, et aussi, par l'affection que Pénélope leur démontre, qu'elle les garderait pour un usage simplement ornemental. ${ }^{49}$

Une autre hypothèse ou conjecture - plus audacieuse et peut-être un peu plus pausible que celle de K. F. Vickery à partir de ses données égyptiennes, mais également invérifiable et déplacée dans l'ensemble des usages de l'Odyssée - est celle d'Andrew Dalby: les oies seraient nourries ou plutôt gavées (ce qu'il déduit de la manière unique de les nourrir) peut-être à cause de leur foie, c'est à dire pour en faire du foie gras. ${ }^{50}$

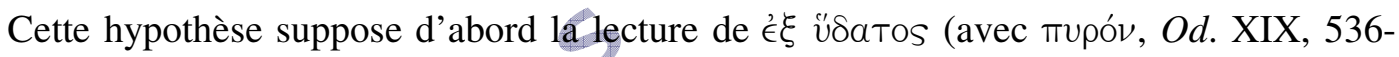
537) que Jaccottet a renvoyée à une note en bas de page (“du grain trempé d'eau”): “ 'I have twenty geese at home, eating wheat soaked in water', says Penelope in the Odyssey passage, beginning to tell her dream", 51 parce que cette lecture renforce le soin spécifique (ou unique) de cette alimentation d'un animal domestique. ${ }^{52}$ Mais, même si -

\footnotetext{
${ }^{49}$ Cf. Vickery, op. cit., p. 67: That the goose was once held sacred fits well with its connection with an omen in the Odyssey where, in Penelope's dream, geese represented the suitors destroyed by the eagle, i.e. Odysseus, and the ornamental character of the Egyptian goose is in keeping both with Penelope's fondness for them and the fact that, apparently, she did keep them for ornamental purposes. Cf. aussi Ameis-Hentze (Homers "Odyssee" für den Schulgebrauch erklärt. Leipzig: Teubner, 1911. Zweiter Band, Zweites Heft, p. 39): 536. (...) Gänse hielt man weniger des Nutzens wegen als zum Vergnügen. Mais on pourrait poser encore la question: quel divertissement alors?

${ }^{50}$ Cf. Dalby, op. cit., p. 64: Geese, "khén", were not only domesticated, they were already being forcefed - and perhaps in particular for the sake of their livers, foie gras. Two quite early references link geese with feeding in a way that seems to set them apart from other farmyard animals ("Odyssey" 19.536-537; Cratinus 49, both cited at Atheneus 384c).

${ }^{51}$ Cf. Dalby, op. cit., p. 64.

${ }^{52}$ Même si A. Dalby n'explicite pas sa liaison avec "la mangeoire" ( $\pi \dot{\in} \in \lambda o \nu$, v. 553) comme le fait Joseph

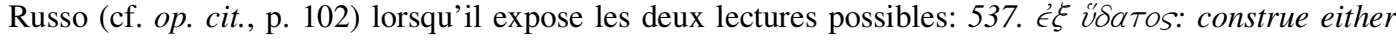

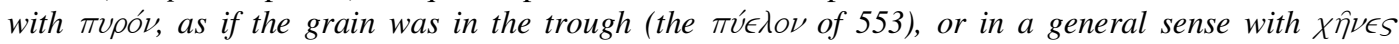

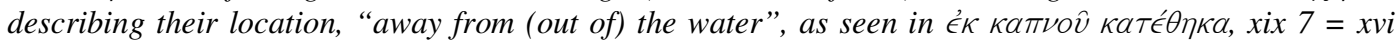
288 (....). Voir aussi ce que disent les scholiaste(s) B. P. de l'Odyssée (Dindorf, G. Scholia Graeca in
} 
d'après les informations d'Andrew Dalby - on la met en rapport avec le gavage par céréales (siteutố) discuté par Athénée (384a-c), ${ }^{53}$ il serait difficile ou impossible de prouver qu'il s'agit déjà dans l'Odyssée du gavage de par la seule présence du "grain trempé d'eau" ou d'une “mangeoire". Si, en plus, à la question du but de ce présumé gavage des oies, on essaie de répondre de manière gastronomique comme si c'était pour le foie gras, le risque de l'anachronisme est encore plus grand, car - selon A. Dalby luimême - la première attestation du foie gras dans la tradition occidentale se trouve dans Horace, ${ }^{54}$ et il n'y a pas du tout un trait quelconque de gastronomie (ou de Delikatessen) dans l'Odyssée, surtout dans les repas ordinaires du monde humain auxquels appartiendraient ces oies de Pénélope. Alors, le maximum qu'on peut imaginer comme usage possible de ces oies de Pénélope (ainsi que de celle plus grande au palais de Ménélas) comme source de nourriture, étant donné aussi la totale inexistence dans l'Odyssée de mention aux œufs de volaille, est celui plus générique de leur viande qui pourrait être reconnue éventuellement comme un plat possible et transposé seulement pour l'auditoire (constituant donc un répertoire indirect et subtil) mais évidemment pas pour les personnages qui dans le monde humain (ou dans des circonstances normales) ne mangent jamais des oiseaux. ${ }^{55}$ Cela nous amène à reconnaître que directement dans le cas de Pénélope les vingt oies qui mangent du blé servent d'abord et surtout comme support d'un symbole onirique - concentré et ambigu - des prétendants gros mangeurs ainsi que des vingt années de sa loyale surveillance de la maison pendant l'absence d'Ulysse. Mais il n'en reste pas moins que ce support ne peut être utilisé que parce qu'il laisse transparaître un arrière-fonds (que les habitudes alimentaires normales de l'Odyssée contredisent) où l'élevage des oies serait difficilement pensable si elles ne pouvaient pas être converties en nourriture (encore que l'hypothèse du foie gras soit trop improbable).

\section{Références}

Homeri “Odysseam”. Oxonii: E Typographeo Academico, 1855. Tomus II, p. 684):

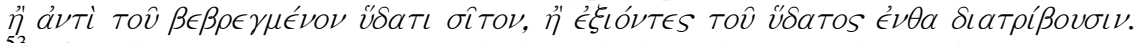

${ }^{53}$ Cf. Dalby, op. cit., p. 224: (...) The force-feeding of geese with cereal ("siteutoi"), which has been the usual method, is discussed by Athenaeus (384a-c): instructions for a Roman readership are given by Cato ("On Agriculture" 89).

${ }^{54}$ Cf. Dalby, op. cit., p. 224: The earliest source in any language that explicitly mentions foie gras as a delicacy is a Roman poet (Horace, Satires 2.8.88).

${ }^{55} \mathrm{On}$ a donc pas besoin là de penser à une quelconque pitié possible de Pénélope pour ses oies en tant que viande mangeable. 
AMEIS, K. F.; HENTZE, C. Homers “Odyssee” für den Schulgebrauch erklärt. Leipzig: Teubner, 1911. Zweiter Band, Zweites Heft.

ATHÉNÉE. Les Deipnosophistes. Texte établi et traduit par Desrousseaux, A.-M. Paris: Les Belles Lettres, 1956. Tome I, livres I et II.

BOBER, P. P. Art, culture and cuisine. Ancient and medieval gastronomy. Chicago: The University of Chicago Press, 1999.

BRUNS, G. Küchenwesen und Mahlzeiten. In: Archaeologia homerica. Göttingen: Vandenhoeck \& Ruprecht, 1970. Band II, Kapitel Q.

CHANTRAINE, P. Dictionnaire étymologique de langue grecque 3-4. Paris: Klincksieck, 1984.

DALBY, A. Siren feasts. A history of food and gastronomy in Greece. London: Routledge, 1996.

DAVIES, M. Feasting and food in Homer. Realism and stylisation. Prometheus. Firenze, fasc. II, p. 97-107, 1997.

DEVEREUX, G. Penelope's character. Psychoanalytic Quarterly. New York, vol. XXVI, p. 378-86, 1957.

DINDORF, W. Scholia Graeca in Homeri "Odysseam”. Oxonii: E Typographeo Academico, 1855. Tomus II.

EBELING, H. (org.). Lexicon homericum. Hildesheim: Georg Olms Verlag, 1987. Vol. II.

ERBSE, H. (org.). Scholia Graeca in Homeri "Iliadem" (Scholia vetera). Berlin: Walter de Gruyter, 1975. Vol. IV.

HARTOG, F. Les Scythes d'Hérodote: le miroir scythe. In: Le miroir d'Hérodote. Essai sur la représentation de l'autre. Paris: Gallimard, 2001, p. 57-67.

HOMÈRE. Iliade. Traduction de Paul Mazon, préface de Pierre Vidal-Naquet. Paris: Gallimard, 1995.

HOMĖRE. L'Odyssée. Traduction et notes de Philippe Jaccottet. Paris: François Maspero, 1982.

HOMERI Odyssea. Recognovit Peter Von der Mühll. Leipzig: Teubner, 1983.

HOMERI opera. Recognoverunt Thomas W. Allen and David B. Monro. Oxford: University Press, 1988-1989. Tomi I-II.

PRATT, L. "Odyssey" 19.535-50: on the interpretation of dreams and signs in Homer. Classical Philology. Chicago, n. LXXXIX, p. 148-53, 1994. 
RICHTER, W. Archaeologia homerica. Göttingen: Vandenhoeck \& Ruprecht, 1968. Band II, Kapitel H.

RUSSO, J. Interview an after-math: dream, fantasy, and intuition in "Odyssey" 19 and 20. American Journal of Philology. Baltimore, n. CIII, p. 4-18, 1982.

RUSSO, J. Books XVII-XX. In: RUSSO, J.; FERNÁNDEZ-GALIANO, M.; HEUBECK, A. A commentary on Homer's “Odyssey”. Oxford: Clarendon Press, 1992. Vol. III, p. 1-127.

SCHMIDT, M. Die Erklärungen zum Weltbild Homers und zur Kultur der Heroenzeit in den b-T Scholien zur "Ilias". Zetemata. München, Heft LXII, 1976.

SNELL, B. (begründet von); MEIER-BRÜGER, M. (Redaktor). Lexicon des frühgriechischen Epos. Göttingen: Vandenhoeck \& Ruprecht, 2004. Band 3.

VICKERY, K. F. Food in early Greece. Illinois Studies in the Social Sciences. Urbana. Vol. XX, n. 3, 1936.

VIDAL-NAQUET, P. Valeurs mythiques et religieuses de la terre et du sacrifice dans l' "Odyssée". In: . Le chasseur noir. Paris: Maspero, 1981, p. 39-68.

WEST, S. Books I-IV. In: HEUBECK, A.; WEST, S.; HAINSWORTH, J. B. A commentary on Homer's “Odyssey”. Oxford: Clarendon Press, 1988. Vol. I, p. 49-245.

RESUME: Cet article présente et commente trois types de nourriture possible (les fruits, les légumes et les oies de Pénélope) qui dans l'Odyssée ne composent jamais le menu d'un repas traditionnel dans le monde humain (c'est-à-dire, la viande rôtie d'animaux domestiques sacrifiables, le pain de blé ou d'orge, et le vin mélangé à l'eau), mais qui pourraient bien être perçus en tant que tels pâr l'auditoire, constituant ainsi dans le poème lui-même - quoique d'une manière indirecte - un répertoire alimentaire plus large qui, par contraste, révélerait la restriction et le choix (très bien marqués) de ce que les personnages mangent dans le monde humain.

MOTS-CLES: nourriture ; Odyssée ; fruits ; légumes; les oies de Pénélope.

ABSTRACT: This article presents and comments about three possible types of food (fruits, vegetables and Penelope's geese) which in the Odyssey never composes the menu in a traditional meal in the human world (that is: grilled meat of domestic animals able to be sacrificed, wheat or barley bread, and wine mixed to water), but which could be noticed as such by the audience, thus constituting within the poem itself - even if indirectly - a wider food repertoire which, by contrast, would reveal the well marked restriction and choice of what the characters eat in the human world.

KEY WORDS: food; Odyssey; fruits; vegetables; Penelope's geese. 